\title{
Advances in In Vivo Imaging Techniques for the Visualization and Quantification of DC-T Cell Interactions
}

\section{Abbirami Sathappan and Nicholas van Panhuys *}

Sidra Medical and Research Center, Doha, Qatar

*Corresponding author: Nicholas van Panhuys, Asthma and Allergy Group, Department of Translational Medicine, Sidra Medical and Research Center, PO Box 26999, Doha, Qatar, Tel: 009744003 7559; E-mail: nvanpanhuys@sidra.org

Received date: May 09, 2017; Accepted date: May 25, 2017; Published date: May 29, 2017

Copyright: ( 2017 van Panhuys N. This is an open-access article distributed under the terms of the Creative Commons Attribution License, which permits unrestricted use, distribution, and reproduction in any medium, provided the original author and source are credited.

Keywords: Two-photon; T-cell; Dendritic cell; Interaction; Intravital microscopy

\section{Short Communication}

Since the first application of two photon intravital microscopy (2PM) to the study of the immune system in 1997 [1], 2PM imaging has offered several major advantages over previous techniques for study immune interactions. Such as 2D plate based culture systems, as cells exhibit significantly different migratory behavior in $2 \mathrm{D}$ as compared to 3D. Whilst, 3D matrigel systems allow cells to migrate in a more natural manner, they fail to adequately replicate a physiological environment which is densely packed with other cell types and contains a myriad of environmental cues such as chemokines, adhesion molecules and lymphatic flow, these factors are largely overcome by the use of ex-vivo/explant organ imaging. However, this system also has significant limitations in that cells cannot be recruited to or exit the organ of interest and due to limitations in tissue oxygenation, imaging sessions can generally only be conducted for less than one hour [2]. Therefore, the use of intravital 2PM has opened up a new era of understanding in immunology. Especially in terms of how we view the importance of the migratory behavior and the interactions that occur between cells of the immune system under both homeostatic and inflammatory conditions.

Perhaps this has been most enlightening when applied to the study of the adaptive immune system and the interaction between CD4+ and CD8+ T cells with dendritic cells (DC). These studies have revealed that $\mathrm{T}$ cells are constantly forming non-cognate interactions with DC as they migrate through the lymph node (LN) at an average velocity of $10 \mu \mathrm{m} / \mathrm{min}$, allowing individual DC to briefly interact with an estimated $5000 \mathrm{~T}$ cells an hour $[3,4]$. This behavior allows for the rapid scanning of the TCR repertoire during an immune response and the swift activation of antigen specific T-cells. Initial cognate interactions consist of brief phase I interactions ( 0-6 h) typically lasting 10-30 minutes each, before progression to phase II interactions where stable immune synapses are formed at $\sim 6-18 \mathrm{~h}$ post-activation, a transition to phase III interactions then occurs between $\sim 18-30 \mathrm{~h}$, which is typified by multiple short lived interactions and a pattern of swarming behavior $[5,6]$. The quality or quantity of the cognate antigen and both the phenotype and activation status of the stimulatory DC have all been shown to influence the process of activation and differentiation $[7,8]$, and the application of novel techniques for probing this process will undoubtedly allow for a much greater understanding of the adaptive immune response.

\section{Tracking with Novel Fluorescent Proteins/Dyes:}

The capabilities and application of intravital 2PM for the analysis of cellular processes and the dynamics of interactions has seen an exponential growth with the introduction of several novel fluorescent proteins, optical markers and NIR (Near Infrared) fluorophores. The use of optical markers such as photoactivatable (PA) [9] fluorescent proteins PA-GFP and photo-convertible fluorophores (e.g. Kaede [10] and kikGR [11]), have become an invaluable tool for the longitudinal imaging of immune responses [12]. These fluorescent proteins are capable of exhibiting prominent changes in their spectral properties in response to light irradiated at a specific wavelength, allowing for their use in labelling specific cells or populations within lymph node microenvironments. The combination of high spatio-temporal resolution, low bleaching and minimal photo-toxicity to cells, makes long term imaging feasible for numerous hours or even days. Studies have demonstrated the use of PA-GFP in precise bulk labeling and migration tracking of $\mathrm{B}$ cells between the dark and light zones within the GCs [13]. Due to the irreversible photo-convertible properties of Kaede, discontinuous tracking of the optically highlighted cells using two photon intravital imaging is possible, where the highlighted cells can be tracked even after they have left the initial imaging volume and migrated to distant LN compartments or organs. Following photoconversion optically highlighted B cells were shown to spread out over the entire B cell compartment within 24 hours of activation, with additional data gained from studying Kaede labelled cells confirming results from previous lymphocyte recirculation studies, indicating that the average lymph node residence time is $12-24$ hours [13]. Hence, the use of the optically highlighted fluorescent proteins in conjugation with 2PM imaging is ideal for migration tracking and fate analysis of large numbers of immune cells from one region of interest to another over several days to weeks.

The brainbow system has additionally proved to be highly useful in the study of immune cell development. Brainbow utilizes a Cre-Lox recombination approach featuring multiple XFP expression sites, in which the protein Cre recombinase drives inversion or excision of DNA between loxP sites, allowing for expression of single or multiple XFPs allowing for the expression of distinct cell lineage tracing colors to be expressed. This multicolor imaging method, traditionally developed to study neural circuits is now being used in studying immune system such as the development of Langerhans cells and follicular dendritic cells and antigen class switching during B cell maturation in the germinal center [14-16], indicating the potential to use the brainbow system for tracking specific T-cell clones or differentiation states.

Currently red and far red fluorescent dyes and proteins are widely used as fluorophores in intravital 2PM. However, a new generation of 
NIR dyes, which allow for deep tissue penetration, minimal photo damage and low background auto fluorescence are currently being developed, including squaraine and squaraine-rotaxanes derivatives, with cross sections of $33,000 \mathrm{GM}$ and molecular weights much smaller than current quantum dots, the results of early studies are proving promising for the development of novel tools for subcellular imaging [17-19]. Additionally, fluorescent proteins [20] and nanotube-based optical sensors [21] that are excitable in the 1000-1300 nm range are being designed to allow for imaging at greater depths than what is achievable with conventional fluorophores.

\section{MP Analysis of Transcriptional Factor Activation and In Vivo Signaling:}

In addition to tracking cellular migration and the dynamics of interactions, several systems have been developed to study the downstream signaling events that occur during $\mathrm{T}$ cell activation. Multiple studies have used intracellular $\mathrm{Ca}^{2+}$ dyes [8] and protein based fluorescent indicators, such as GCamp [22] to study the release of calcium as a proximal indicator of activation. 2MP imaging can also be used in vivo to study the translocation of transcriptional regulators during immune responses. Marangoni et al. [23] reported expression of a modified NFAT1-GFP in combination with H2B-RFP in CD4+ T cells led to the determination of the relative time differences in nuclear import and export of NFAT into the nucleus during activation in both lymph node and tumor environments. Here, NFAT nuclear export was found to have a $t 1 / 2$ of 20 minutes indicating that this temporary period of NFAT recruitment may act as a mechanism of transcriptional memory, capable of recording previous interactions and adding them in an iterative fashion $[23,24]$. Additional, studies assessing other proximal measures of TCR activation have relied on the use of a semiautomated quantification methodology to assess PKCל-GFP and LATGFP activity following retroviral expression. In order to study the subcellular distribution of fluorescent fusion proteins in cells, migrating within tissues. Here, GFP-PKC $\zeta$ was found to accumulated in the rear end of the migrating thymocytes and researchers further observed a differential distribution of LAT-GFP within T-cells [25]. Additionally, transgenic biosensor models for measuring cellular activation through the expression of FRET based biosensors for PKA [26] and ERK [27] have also been developed.

Dynamic in situ cytometry (DISC), represents a readily accessible method for the use of $2 \mathrm{PM}$ imaging for studying in vivo activation and for phenotypic analysis [28]. By utilizing the in vivo antibody labeling of CD4+ $\mathrm{T}$ cells with $\mathrm{CD} 62 \mathrm{~L}$ and then tracking $\mathrm{T}$ cell dynamic interactions with DC, TCR signaling was assessed by analysing shedding of CD62L. Here, CD62L shedding was found to be a marker of early TCR signaling, allowing for the examination of how $\mathrm{T}$ cells sense TCR ligands of varying affinities in vivo [28]. A similar technique has also been applied by other researchers to study blood and lymph vessels during intravital imaging [29].

Recent advancements in optogenetic tools with ion sensitivity in near-infrared regions have been developed for wireless modulation of immune responses and cellular immunotherapy. Peter et al. [30] recently engineered a complex consisting of an opto- $\mathrm{Ca}^{2+}$ release activated calcium channel (opto-CRACs) coupled to an up conversion nanoparticle (UCNP) doped with lanthanide. Whereby the UCNPs act as nano-transducers, converting tissue penetrating NIR into visible light emission for the remote activation of opto-CRACs [30,31], allowing for cellular activation of the $\mathrm{Ca}^{2+}$ secondary messenger system. Imaging applications of the opto-optoCRACs systems are multifold, including the modulation of calcium dependent $\mathrm{T}$ cell activation, DC maturation and modulation of antigen presentation and the activation of inflammatory pathways [30,31].

The application of $2 \mathrm{PM}$ imaging to the study of in vivo immune interactions has already led to major insights into the dynamics of cellular behavior during the activation of T cells by DCs. Future studies will undoubtedly benefit from the advent of the range of new technologies described here allowing investigators to probe additional characteristics of activation, by quantifying single cell signaling, analyzing cell behavior in novel lymph node areas and by tracking these cells for extended periods of time.

\section{Acknowledgement}

Abbirami Sathappan and Nicholas van Panhuys are funded by Sidra Medical and Research Center.

\section{References}

1. Ingulli E, Mondino A, Khoruts A, Jenkins MK (1997) In vivo detection of dendritic cell antigen presentation to CD4(+) T cells. J Exp Med 185: 2133-2141.

2. Weigert R, Porat-Shliom N, Amornphimoltham P (2013) Imaging cell biology in live animals: ready for prime time. J Cell Biol 201: 969-979.

3. von Andrian UH, Mempel TR (2003) Homing and cellular traffic in lymph nodes. Nat Rev Immunol 3: p. 867-878.

4. Miller MJ, Hejazi AS, Wei SH, Cahalan MD, Parker I (2004) T cell repertoire scanning is promoted by dynamic dendritic cell behavior and random T cell motility in the lymph node. Proc Natl Acad Sci U S A 101: 998-1003.

5. Henrickson SE, Mempel TR, Mazo IB, Liu B, Artyomov MN, et al. (2008) In vivo imaging of T cell priming. Sci Signal 1: pt2.

6. Mempel TR, Henrickson SE, Von Andrian UH (2004) T-cell priming by dendritic cells in lymph nodes occurs in three distinct phases. Nature 427: 154-159.

7. Henrickson SE, Mempel TR, Mazo IB, Liu B, Artyomov MN et al. (2008) $T$ cell sensing of antigen dose governs interactive behavior with dendritic cells and sets a threshold for T cell activation. Nat Immunol 9: 282-291.

8. van Panhuys N, Klauschen F, Germain RN (2014) T-Cell-ReceptorDependent Signal Intensity Dominantly Controls CD4(+) T Cell Polarization In Vivo. Immunity 41: 63-74.

9. Patterson GH, Lippincott-Schwartz J (2002) A photoactivatable GFP for selective photolabeling of proteins and cells. Science 297: 1873-1877.

10. Ando R, Hama H, Yamamoto-Hino M, Mizuno H, Miyawaki A (2002) An optical marker based on the UV-induced green-to-red photoconversion of a fluorescent protein. Pro Natl Acad Sci USA 99: 12651-12656.

11. Tsutsui H, Karasawa S, Shimizu H, Nukina N, Miyawaki A (2005) Semirational engineering of a coral fluorescent protein into an efficient highlighter. ЕMBO Rep 6: 233-238.

12. Lukyanov KA, Chudakov DM, Lukyanov S, Verkhusha VV (2005) Innovation: Photoactivatable fluorescent proteins. Nat Rev Mol Cell Biol 6: 885-890.

13. Victora GD, Schwickert TA, Fooksman DR, Kamphorst AO, MeyerHermann M, et al. (2011) Germinal Center Dynamics Revealed by Multiphoton Microscopy with a Photoactivatable Fluorescent Reporter. Cell 143: 592-605.

14. Ghigo C, Mondor I, Jorquera A, Nowak J, Wienert S, et al. (2013) Multicolor fate mapping of Langerhans cell homeostasis. J Exp Med 210: 1657-1664.

15. Jarjour M, Jorquera A, Mondor I, Wienert S, Narang P, et al. (2014) Fate mapping reveals origin and dynamics of lymph node follicular dendritic cells. J Exp Med 211: 1109-1122. 
Citation: Sathappan A, van Panhuys N (2017) Advances in In Vivo Imaging Techniques for the Visualization and Quantification of DC-T Cell Interactions. J Immuno Biol 2: 125. doi:10.4172/2476-1966.1000125

Page 3 of 3

16. Snippert HJ, van der Flier LG, Sato T, van Es JH, van den Born M, et al. (2010) Intestinal Crypt Homeostasis Results from Neutral Competition between Symmetrically Dividing Lgr5 Stem Cells. Cell 143: 134-144.

17. Podgorski K, Terpetschnig E, Klochko OP, Obukhova OM, Haas K (2012) Ultra-bright and -stable red and near-infrared squaraine fluorophores for in vivo two-photon imaging. PloS one 7: e51980-e51980.

18. Chung SJ, Zheng S, Odani T, Beverina L, Fu J, et al. (2006) Extended Squaraine Dyes with Large Two-Photon Absorption Cross-Sections. J Amer Chem Soc 128: 14444-14445.

19. Guo Z, Park S, Yoon J, Shin I (2014) Recent progress in the development of near-infrared fluorescent probes for bioimaging applications. Chem Soc Rev 43: m16-29.

20. Tischbirek CH, Birkner A, Konnerth A (2015) In vivo deep two-photon imaging of neural circuits with the fluorescent Ca2+ indicator Cal-590. J Physiol.

21. Roxbury D, Jena PV, Williams RM, Enyedi B, Niethammer P, et al, (2015) Hyperspectral Microscopy of Near-Infrared Fluorescence Enables 17Chirality Carbon Nanotube Imaging. Sci Rep 5: 14167.

22. Yoshikawa S, Usami T, Kikuta J, Ishii M, Sasano T, et al, ( 2016)Intravital imaging of $\mathrm{Ca}(2+)$ signals in lymphocytes of $\mathrm{Ca}(2+)$ biosensor transgenic mice: indication of autoimmune diseases before the pathological onset. Sci Rep 6: 18738.

23. Marangoni F, Murooka TT, Manzo T, Kim EY, Carrizosa E, et al, (2013) The transcription factor NFAT exhibits signal memory during serial $\mathrm{T}$ cell interactions with antigen-presenting cells. Immunity 38: 237-249.
24. Lodygin D, Odoardi F, Schläger C, Körner H, Kitz A, et al, (2013) A combination of fluorescent NFAT and H2B sensors uncovers dynamics of $\mathrm{T}$ cell activation in real time during CNS autoimmunity. Nature Medicine 19: 784-790

25. Melichar HJ, Li O, Herzmark P, Padmanabhan RK, Oliaro J, et al, (2011) Quantifying subcellular distribution of fluorescent fusion proteins in cells migrating within tissues. Immunology and Cell Biology 89: 549-557.

26. Kamioka Y, Sumiyama K, Mizuno R, Sakai Y, Hirata E, et al. (2012) Live imaging of protein kinase activities in transgenic mice expressing FRET biosensors. Cell Struct Funct 37: 65-73.

27. Hiratsuka T, Fujita Y, Naoki H, Aoki K, Kamioka Y, et al, (2015) Intercellular propagation of extracellular signal-regulated kinase activation revealed by in vivo imaging of mouse skin. Elife 4: e05178.

28. Moreau HD, Lemaître F, Terriac E, Azar G, Piel M, et al., Dynamic In Situ Cytometry Uncovers T Cell Receptor Signaling during Immunological Synapses and Kinapses In Vivo. Immunity 37: 351-363.

29. Kilarski WW, Güç E, Teo JC, Oliver SR, Lund AW, et al, (2013) Intravital immunofluorescence for visualizing the microcirculatory and immune microenvironments in the mouse ear dermis. PLoS One 8: e57135.

30. Hegemann P, Möglich A (2011) Andreas, Channelrhodopsin engineering and exploration of new optogenetic tools. Nat Methods 8: 39-42.

31. He L, Zhang Y, Ma G, Tan P, Li Z, et al, (2015) Near-infrared photoactivatable control of $\mathrm{Ca}(2+)$ signaling and optogenetic immunomodulation 4: e10024. 